\title{
SECAPR - A bioinformatics pipeline for the rapid and user- friendly processing of targeted enriched Illumina sequences, from raw reads to alignments
}

\author{
Tobias Andermann ${ }^{\text {Corresp.., }}{ }^{1,2}$ ， Ángela Cano ${ }^{2,3}$, Alexander Zizka ${ }^{1,2}$, Christine Bacon ${ }^{1,2}$, Alexandre Antonelli ${ }^{1,2,4,5}$ \\ 1 Department of Biological and Environmental Sciences, University of Gothenburg, Gothenburg, Sweden \\ 2 Gothenburg Global Biodiversity Centre, Gothenburg, Sweden \\ 3 Department of Botany and Plant Biology, University of Geneva, Geneva, Switzerland \\ 4 Gothenburg Botanical Garden, Gothenburg, Sweden \\ 5 Department of Organismic and Evolutionary Biology, Harvard University, Cambridge, MA, United States \\ Corresponding Author: Tobias Andermann \\ Email address: tobias.andermann@bioenv.gu.se
}

Evolutionary biology has entered an era of unprecedented amounts of DNA sequence data, as new sequencing platforms such as Massive Parallel Sequencing (MPS) can generate billions of nucleotides within less than a day. The current bottleneck is how to efficiently handle, process, and analyze such large amounts of data in an automated and reproducible way. To tackle these challenges we introduce the Sequence Capture Processor (SECAPR) pipeline for processing raw sequencing data into multiple sequence alignments for downstream phylogenetic and phylogeographic analyses. SECAPR is userfriendly and we provide an exhaustive empirical data tutorial intended for users with no prior experience with analyzing MPS output. SECAPR is particularly useful for the processing of sequence capture (synonyms: target or hybrid enrichment) datasets for nonmodel organisms, as we demonstrate using an empirical sequence capture dataset of the palm genus Geonoma (Arecaceae). Various quality control and plotting functions help the user to decide on the most suitable settings for even challenging datasets. SECAPR is an easy-to-use, free, and versatile pipeline, aimed to enable efficient and reproducible processing of MPS data for many samples in parallel. 


\section{Author Cover Page}

2 Article submission to PeerJ

3 Manuscript category: Bioinformatics Tool

4 Collection: "Endless forms: Advances in evolutionary analyses of biodiversity"

5 Article title: SECAPR - A bioinformatics pipeline for the rapid and user-friendly processing 6 of targeted enriched Illumina sequences, from raw reads to alignments

8 Authors: Tobias Andermann* ${ }^{(1,2)}$, Ángela Cano ${ }^{(2,3)}$, Alexander Zizka ${ }^{(1,2)}$, Christine Bacon ${ }^{(1,2)}$,

9 Alexandre Antonelli $(1,2,4,5)$

11 Affiliations:

$12{ }^{1}$ Department of Biological and Environmental Sciences, University of Gothenburg, Box 461, SE

13405 30, Göteborg, Sweden

$14{ }^{2}$ Gothenburg Global Biodiversity Centre, Göteborg, 41319, Sweden

$15{ }^{3}$ Department of Botany and Plant Biology, University of Geneva, Geneva, Switzerland

$16{ }^{4}$ Gothenburg Botanical Garden, Göteborg, 41319, Sweden

$17{ }^{5}$ Department of Organismic and Evolutionary Biology, Harvard University, Cambridge, MA 1802138 USA 


\section{Abstract}

22 Evolutionary biology has entered an era of unprecedented amounts of DNA sequence data, as 23 new sequencing platforms such as Massive Parallel Sequencing (MPS) can generate billions of 24 nucleotides within less than a day. The current bottleneck is how to efficiently handle, process, 25 and analyze such large amounts of data in an automated and reproducible way. To tackle these 26 challenges we introduce the Sequence Capture Processor (SECAPR) pipeline for processing raw 27 sequencing data into multiple sequence alignments for downstream phylogenetic and 28 phylogeographic analyses. SECAPR is user-friendly and we provide an exhaustive empirical 29 data tutorial intended for users with no prior experience with analyzing MPS output. SECAPR is 30 particularly useful for the processing of sequence capture (synonyms: target or hybrid

31 enrichment) datasets for non-model organisms, as we demonstrate using an empirical sequence 32 capture dataset of the palm genus Geonoma (Arecaceae). Various quality control and plotting 33 functions help the user to decide on the most suitable settings for even challenging datasets.

34 SECAPR is an easy-to-use, free, and versatile pipeline, aimed to enable efficient and 35 reproducible processing of MPS data for many samples in parallel. 


\section{Introduction}

37 An increasing number of studies apply sequence data generated by Massive Parallel Sequencing

38 (MPS) to answer phylogeographic and phylogenetic questions (e.g. Botero-Castro et al. 2013;

39 Smith et al. 2014; Faircloth et al. 2015; Heyduk et al. 2016). Researchers often decide to

40 selectively enrich and sequence specific genomic regions of interest, rather than sequencing the

41 complete genome. One reason is that enriching specific markers leads to a higher sequencing

42 depth for each individual marker, as compared to the alternative of sequencing full genomes.

43 Sequencing depth is important for the extraction of single nucleotide polymorphisms (SNPs) and

44 for allele phasing (Andermann et al., 2018; Bravo et al., 2018). Additionally, phylogenetic

45 analysis software usually relies on multiple sequence alignments (MSAs) with homologous

46 sequences across many taxa, which are simple to recover when specifically enriching these

47 sequences across all samples prior to sequencing.

48 The enrichment of specific genomic regions (markers) is usually achieved through sequence

49 capture (synonyms: hybrid enrichment, hybrid selection, exon capture, target capture) prior to

50 sequencing (Gnirke et al., 2009). This technique applies specific RNA baits, which hybridize with the target regions and can be captured with magnetic beads. Sequence capture is gaining popularity, as more bait sets for non-model organisms are being developed. Some bait sets are designed to match one specific taxonomic group (e.g. Heyduk et al. 2016; Kadlec et al. 2017), while others are designed to function as more universal markers to capture homologous sequences across broad groups of taxa (e.g. UCEs, Faircloth et al. 2012). After enrichment of targeted markers with such bait sets, the enriched sequence libraries are sequenced on a MPS machine (see Reuter, Spacek, and Snyder 2015).

Despite recent technological developments, analyzing sequencing results is a great challenge due to the amount of data produced by MPS machines. An average dataset often contains dozens to hundreds of samples, each with up to millions of sequencing reads. Such amounts of sequence data require advanced bioinformatics skills for storing, quality checking, and processing the data, which may represent an obstacle for many students and researchers. This bottleneck calls for streamlined, integrative and user-friendly pipeline solutions.

64 To tackle these challenges, here we introduce the Sequence Capture Processor (SECAPR)

65 pipeline, a semi-automated workflow to guide users from raw sequencing results to cleaned and 
66 filtered multiple sequence alignments (MSAs) for phylogenetic and phylogeographic analyses.

67 We designed many of the functionalities of this pipeline toward sequence capture datasets in

68 particular, but it can be effectively applied to any MPS dataset generated with Illumina sequencing (Illumina Inc., San Diego, CA, USA). SECAPR comes with a detailed documentation in form of an empirical data tutorial, which is explicitly written to guide users with no previous experience with MPS datasets. To simplify the processing of big datasets, all available functions are built to process batches of samples, rather than individual files. We developed SECAPR to provide the maximum amount of automation, while at the same time allowing the user to choose appropriate settings for their specific datasets. The pipeline provides several plotting and quality-control functions, as well as more advanced processing options such as the assembly of fully phased allele sequences for diploid organisms (Andermann et al., 2018).

\section{Material \& Methods}

The SECAPR pipeline in a nutshell

SECAPR is a platform-independent pipeline written in python, and tested for full functionality on Linux and MacOS. It can be easily downloaded together with all its dependencies as a virtual environment, using the conda package manager (see Availability). The strength of SECAPR is that it channels the main functionalities of many commonly used bioinformatics programs and enables the user to apply these to sets of samples, rather than having to apply different software to each sample individually. In addition, SECAPR is optimized for high performance computing as it enables computational parallelization for all major functions, which allows the efficient processing of datasets encompassing dozens to hundreds of samples in parallel.

The basic SECAPR workflow (Fig. 1) includes the following steps:

\section{Quality filtering and adapter trimming}

2. De novo contig assembly

3. Selection of target contigs

4. Building MSAs from contigs

5. Reference-based assembly 
96

SECAPR automatically writes summary statistics for each processing step and sample to a logfile (summary_stats.txt, Table 1). The pipeline includes multiple visualization options to gauge data quality and, if necessary, adapt processing settings accordingly. SECAPR comes with a detailed documentation and data tutorial (see Availability).

\section{Description of the SECAPR workflow}

1. Quality filtering and adapter trimming (secapr clean_reads). The SECAPR clean_reads function applies the software Trimmomatic (Bolger, Lohse \& Usadel, 2014) for removing adapter contamination and low quality sequences from the raw sequencing reads (FASTQformat). An additional SECAPR plotting function summarizes FASTQC

(BabrahamBioinformatics) quality reports of all files and produces a visual overview of the whole dataset (Fig. 2). This helps to gauge if the files are sufficiently cleaned or if the clean_reads function should be rerun with different settings.

2. De novo contig assembly (secapr assemble_reads). The SECAPR function assemble_reads assembles overlapping FASTQ reads into longer sequences (de novo contigs) by implementing the de novo assembly software Abyss (Simpson et al., 2009). Abyss has been identified as one of the best-performing DNA sequence assemblers currently available (Hunt et al., 2014). As an alternative to Abyss we also implemented the option to use the Trinity assembler (Grabherr et al., 2011), which currently is only supported for the Linux distribution of SECAPR. We do however recommend the use of Abyss as the preferred assembly-software, firstly due to significantly faster computation and secondly and more importantly due to the fact that Trinity is intended for the assembly of RNA transcriptome data, leading to different assumptions about the input data in comparison to DNA assemblers such as Abyss, as discussed in Haas et al. (2013).

\section{Selection of target contigs (secapr find_target_contigs). The SECAPR function}

find_target_contigs identifies and extracts those contigs that represent the DNA targets of interest. This function implements the program LASTZ (formerly BLASTZ, Harris 2007) by searching the contig files for matches with a user-provided FASTA-formatted reference library. For sequence capture datasets, a suitable reference library is the reference file that was used for 
124 synthesizing the RNA baits, which will return all contigs that match the enriched loci of interest.

125 The find_target_contigs function identifies potentially paralogous loci (loci that have several

126 matching contigs) and excludes these from further processing. It further allows the user to keep

127 or exclude long contigs that match several adjacent reference loci, which can occur if the

128 reference file contains sequences that are located in close proximity to each other on the genome

129 (e.g. several separate exons of the same gene).

130 4. Building MSAs from contigs (secapralign_sequences). The SECAPR function

131 align_sequences builds multiple sequence alignments (MSAs) from the target contigs that were

132 identified in the previous step. The function builds a separate MSA for each locus with matching 133 contigs for $\geq 3$ samples.

134 5. Reference-based assembly (secapr reference_assembly). The SECAPR reference_assembly

135 function applies the BWA mapper (Li \& Durbin, 2010) for reference-based assembly of FASTQ

136 reads and Picard (broadinstitute.github.io/picard/) for removing duplicate reads. The function

137 saves the assembly results as BAM files (Fig 3), transforms them into Variant Call Format

138 (VCF) format using SAMtools (Li et al., 2009), and generates a consensus sequence from the

139 read variation at each locus. These consensus sequences have several advantages over the de

140 novo contig sequences (see Discussion) and can be used for building MSAs with the SECAPR

141 align_sequences function.

142 The reference_assembly function includes different options for generating a reference library for 143 all loci of interest:

144 - -reference_type alignment-consensus: The user provides a link to a folder containing 145 MSAs, e.g. the folder with the contig MSAs from the previous step, and the function 146 calculates a consensus sequence from each alignment. These consensus sequences are then used as the reference sequence for the assembly. This function is recommended when running reference-based assembly for groups of closely related samples (e.g. samples from the same genus or family).

- --reference_type sample-specific: From the MSAs, the function extracts the contigs for each sample and uses them as a sample-specific reference library. If the user decides to use this function it is recommended to only use alignments for reference that contain 
sequences for all samples. This will ensure that the same loci are being assembled for all samples.

- --reference_type user-ref-lib: The user can provide a FASTA file containing a custom reference library.

An additional SECAPR function (locus_selection) allows the user to select a subset of the data consisting of only those loci, which have the best read-coverage across all samples. sort out the two phases (reads covering different alleles) at a given locus. This function applies the phasing algorithm as implemented in SAMtools, which uses read connectivity across multiple variable sites to determine the two phases of any given diploid locus (He et al., 2010). After running the phasing algorithm, the phase_alleles function outputs a separate BAM-file for each allele and generates consensus sequences from these allele BAM-files. This results into two sequences at each locus for each sample, all of which are collected in one cumulative sequence file (FASTA). This sequence file can be run through the SECAPR align_sequences function in order to produce MSAs of allele sequences.

7. Pipeline automation (secaprautomate_all). SECAPR provides the automate_all function that produces contig MSAs and phased allele MSAs from cleaned FASTQ files, only requiring one single command, automating steps 2-6. The user can choose between three different settings, namely relaxed, medium or conservative, which run the SECAPR pipeline with different sensitivity parameters. The relaxed setting is recommended when samples in the dataset are expected to show considerable genetic variation (e.g. samples from different families) or are expected to differ considerably from the provided reference library used for the extraction of target contigs. The conservative setting on the other hand uses very restrictive similarity thresholds for the identification of target contigs and during reference-based assembly and is therefore recommended for datasets containing closely related samples (within same genus) that are expected to also be similar to the provided reference library. The medium setting constitutes an intermediate between these two extremes and is recommendable for datasets, which contain samples of closely related genera. While in all cases it is recommendable to run the individual SECAPR functions with customized settings for each specific dataset, the automate_all function can help to get a first impression of the dataset through the SECAPR-logged sample information 
183 (Table 1) and the inspection of the produced MSAs and other, intermediate files, such as the 184 reference-assembly BAM-files.

Benchmarking with empirical data

187 We demonstrate the functionalities of SECAPR on a novel dataset of target sequencing reads of

188 Geonoma, one of the most species-rich palm genera (plant family Arecaceae) of tropical Central 189 and South America (Dransfield et al., 2008) (Henderson, 2011). Our data comprise newly 190 generated Illumina sequence data for 17 samples of 14 Geonoma species (Supplementary Table

191 S1), enriched through sequence capture. The bait set for sequence capture was designed

192 specifically for palms by Heyduk et al. (2016) to target 176 genes with in total 837 exons. More 193 detailed information about the generation of the sequence data can be found in Appendix 1

194 (Supplemental Material). All settings and commands used during processing of the sequence 195 data can be found in the SECAPR documentation on our GitHub page (see Availability). An 196 example of a downstream application of the MSAs produced by SECAPR for phylogeny 197 estimation can be found in Appendix 2 (Supplemental Material).

\section{Results}

200

The newly generated Geonoma data used for benchmarking constitute an empirical example of a challenging dataset, characterized by irregular read coverage and multiple haplotypes. Despite these challenges, the SECAPR workflow provides the user all the necessary functions to filter and process datasets into MSAs for downstream phylogenetic analyses.

After de novo assembly (secapr assemble_reads) we recovered an average of 323 (stdev=14) contigs per sample (secapr find_target_contigs) that matched sequences of the 837 targeted exons (Table 1, Fig 4a, Supplementary Table S2). In total 45 exons were recovered for all samples. Many of the recovered target contigs spanned several reference exons (all samples: mean $=100$, stdev $=25$ ) and hence were flagged as contigs matching multiple loci (Supplementary Table S3). Since these contigs may be phylogenetically valuable, as they contain the highly variable interspersed introns, we decided to keep these sequences. We extracted these longer

211 contigs together with all other non-duplicated contigs that matched the reference library (secapr 
212 find_target_contigs) and generated MSAs for each locus that could be recovered in at least three

213 Geonoma samples (secapralign_sequences). This resulted in contig alignments for 593 exon

214 loci (Fig. 4a).

215 During reference-based assembly (secapr reference_assembly) we mapped the reads against the

216 consensus sequence of the contig MSAs for all loci. We found an average of 439 exon loci

$217($ stdev $=82)$ per sample that were covered by more than three reads (average coverage across

218 complete locus, Fig. 4b). Hence, our approach of mapping FASTQ reads to libraries compiled

219 from the data leads to an increase of recovered loci per sample, from 323 resulting from de novo

220 assembly to 439 from the referenced-based assembly (36\% increase). Further, the number of loci

221 that were recovered with sufficient coverage for all samples increased by $116 \%$, from 45 after

222 the de novo assembly, to 97 after the reference-based assembly (Supplementary Table S4). We

223 extracted the 50 loci with the best coverage across all samples (secapr locus_selection), as

224 shown in Figs. 4c and 4d. In cases of irregular read-coverage across samples (as in our sample

225 Geonoma data), we strongly recommend the use of the locus_selection function before further

226 processing the data, as demonstrated in our tutorial (see Availability).

227 The results of the reference-based assembly also revealed that our sample data showed more than

228 two haplotypes for many loci. Future research may clarify whether this is the result of various

229 paralogous loci in the dataset or if our Geonoma samples are polyploid due to a recent genome

230 duplication or hybridization event in the ancestry of the genus. Due to the presence of more than

231 two haplotypes at various loci, the results of the allele-phasing step (secapr phase_alleles) are to

232 be viewed critically, since the algorithm is built for phasing the read data of diploid organisms or

233 loci only. All phased BAM files and the compiled allele MSAs are available online (see

234 Availability).

236 Discussion

237 De novo assembly vs. reference-based assembly

238 There are several ways of generating full sequences from raw FASTQ-formatted sequencing 239 reads. The SECAPR pipeline contains two different approaches, namely de novo assembly and

240 reference-based assembly (Fig. 1). De novo assembly can be directly applied to any raw read 
241 data while reference-based assembly requires the user to provide reference sequences for the

242 assembly. We find for the Geonoma example data that reference-based assembly results into

243 recovering more target sequences per sample (Fig. 4d) and provides the user a better handle on

244 quality and coverage thresholds. It is also computationally much less demanding in comparison

245 to de novo assembly.

246 However, reference-based assembly is very sensitive toward the user providing orthologous

247 reference sequences that are similar enough to the sequencing reads of the studied organisms. If

248 the reference sequences are too divergent from the sequenced organisms, only a small fraction of

249 the existing orthologous sequencing reads will be successfully assembled for each locus. In

250 contrast, when relaxing similarity thresholds and other mapping parameters too much (e.g. to

251 increase the fraction of reads included in the assembly) there is higher a risk of assembling non-

252 orthologous reads, which can lead to chimeric sequences being assembled. This can be a

253 problem, particularly in cases of datasets containing non-model organisms, since suitable

254 reference sequences for all loci usually do not exist.

255 For this reason, the SECAPR workflow encourages the user to use these two different assembly

256 approaches in concert (Fig. 1). Our general suggestion is to first assemble contig MSAs for all

257 regions of interest, resulting from de novo assembly and then use these MSAs to build a

258 reference library for reference-based assembly. In that case SECAPR produces a reference

259 library from the sequencing data itself, which is specific for the taxonomic group of interest or

260 even for the individual sample.

261 A common approach is to stop data processing after the de novo assembly step and then use the

262 contig MSAs for phylogenetic analyses (e.g. Faircloth et al. 2012; B. T. Smith et al. 2014;

263 Faircloth 2015). Here we take additional processing steps, including generating new reference

264 libraries for all samples and using these for reference-based assembly. There may be several

265 reasons for carrying out these additional steps:

266 1. Sensitivity: In order to identify de novo contigs that are orthologous to the loci of interest, the user is usually forced (because of the lack of availability) to use a set of reference sequences for many or all loci that are not derived from the studied group. Additionally these reference sequences may be more similar to some sequenced samples than to others, which can introduce a bias in that the number of recovered target loci per sample 
271

272

273

274

275

276

277

278

279

280

281

282

283

284

285

286

287

288

289

290

291

292

293

294

295

296

297

298

299

300

is based on how divergent their sequences are to the reference sequence library. In other words, the 'one size fits all' approach for recovering contig sequences is not the preferred option for most datasets and may lead to taxonomic biases. For this reason it is recommended to generate family, genus or even sample-specific reference libraries using the recovered contigs, and use these to re-assemble the sequencing reads.

2. Intron/exon structure: Another reason for creating a new reference library from the data is that available reference sequences often constitute exons, omitting the interspersed intron sequences (as in the case of using bait sequences as the reference library). The more variable introns in between exons are usually not suitable for designing baits, they are too variable, but are extremely useful for most phylogenetic analyses because they have more parsimony informative sites. There is a good chance that the assembled contigs will contain parts of the trailing introns or even span across the complete intron, connecting two exon sequences (e.g. Bi et al. 2012). This is why it is preferable to use these usually longer and more complete contig sequences for reference-based assembly, rather than the shorter exon sequences from the bait sequence file, in order to capture all reads that match either the exon or the trailing intron sequences at a locus.

3. Allelic variation: Remapping the reads in the process of reference-based assembly will identify the different allele sequences at a given locus. This can also aid in the evaluation of the ploidy level of samples and in identifying loci potentially affected by paralogy.

4. Coverage: Reference-based assembly will give the user a better and more intuitive overview over read-depth for all loci. There are excellent visualization softwares (such as Tablet, by Milne et al. 2013) that help interpret the results.

\section{Novelty}

Several pipelines and collections of bioinformatics tools exist for processing sequencing reads generated by MPS techniques, e.g. PHYLUCE (Faircloth, 2015), GATK (McKenna et al., 2010) and 'reads2trees' (Heyduk et al., 2016). In contrast to some of these existing pipelines, SECAPR i) is targeted towards assembling full sequence data (as compared to only SNP data, e.g. GATK); ii) is intended for general use (rather than project specific, e.g. reads2trees); iii) is optimized particularly for non-model organisms and non-standardized sequence capture datasets (as 
301 compared to specific exon sets, e.g. PHYLUCE); iv) allows allele phasing and selection of the

302 best loci based on read coverage, which to our knowledge are novel to SECAPR. This is possible

303 due to the approach of generating a clade- or even sample-specific reference library from the

304 sequencing read data, which is then used for reference-based assembly; v) offers new tools and

305 plotting functions to give the user an overview of the sequencing data after each processing step.

306

307 Conclusions

308 The SECAPR pipeline described here constitutes a bioinformatic tool for the processing and

309 alignment of raw Illumina sequence data. It is particularly useful for sequence capture datasets

310 and we show here how it can be applied to even challenging datasets of non-model organisms.

\section{Acknowledgements}

313 We thank Goo Jun, Corinne Grover and one anonymous reviewer for valuable feedback on

314 earlier drafts of this manuscript. Further, we thank Estelle Proux-Wéra and Marcel Martin at the

315 National Bioinformatics Infrastructure Sweden at SciLifeLab for their support with turning the

316 SECAPR pipeline into a functioning conda package and for additional support in software

317 development questions. The code for some of the functions of the SECAPR pipeline is inspired

318 from similar functions in the PHYLUCE pipeline (Faircloth, 2015).

319

320 Availability

321 The SECAPR pipeline is open source and freely available from

322 http://www.github.com/AntonelliLab/seqcap_processor. SECAPR and all software dependencies

323 can be downloaded as a virtual environment with the conda package manager

324 (http://bioconda.github.io/recipes/secapr/README.html). Installation instructions, a detailed

325 documentation and an empirical data tutorial with the Geonoma sample data can be found at

326 http://github.com/AntonelliLab/seqcap processor/blob/master/documentation.ipynb. The raw

327 sequencing data for all Geonoma samples is available at 
328 https://www.ncbi.nlm.nih.gov/sra/SRP131660. All other empirical data produced in this study is

329 available from Zenodo (https://doi.org/10.5281/zenodo.1162653).

330

331

332

333

334

335

336

337

338

339

340

341

342

343

344

345

346

347

348

349

350

351

352

353

354

355

\section{References}

Andermann T., Fernandes AM., Olsson U., Topel M., Pfeil B., Oxelman B., Aleixo A., Faircloth BC., Antonelli A. 2018. Allele Phasing Greatly Improves the Phylogenetic Utility of Ultraconserved Elements. bioRxiv:255752. DOI: 10.1101/255752.

BabrahamBioinformatics.FastQC A Quality Control tool for High Throughput Sequence Data. Available at http://www.bioinformatics.babraham.ac.uk/projects/fastqc/ (accessed June 2, 2016).

Bi K., Vanderpool D., Singhal S., Linderoth T., Moritz C., Good JM. 2012. Transcriptome-based exon capture enables highly cost-effective comparative genomic data collection at moderate evolutionary scales. BMC Genomics 13:403. DOI: 10.1186/1471-2164-13-403.

Bolger AM., Lohse M., Usadel B. 2014. Trimmomatic: a flexible trimmer for Illumina sequence data. Bioinformatics 30:2114-20. DOI: 10.1093/bioinformatics/btu170.

Botero-Castro F., Tilak MK., Justy F., Catzeflis F., Delsuc F., Douzery EJP. 2013. Nextgeneration sequencing and phylogenetic signal of complete mitochondrial genomes for resolving the evolutionary history of leaf-nosed bats (Phyllostomidae). Molecular Phylogenetics and Evolution 69:728-739. DOI: 10.1016/j.ympev.2013.07.003.

Bravo GA., Antonelli A., Bacon CD., Bartoszek K., Blom M., Huynh S., Jones G., Knowles LL., Lamichhaney S., Marcussen T., Morlon H., Nakhleh L., Oxelman B., Pfeil B., Schliep A., Wahlberg N., Werneck F., Wiedenhoeft J., Willows-Munro S., Edwards S V. 2018. Embracing heterogeneity: Building the Tree of Life and the future of phylogenomics. DOI: 10.7287/peerj.preprints.26449v3.

Dransfield J., Uhl N., Asmussen C., Baker W. 2008. Genera palmarum. Royal Botanic Gardens:410-42.

Faircloth BC. 2015. PHYLUCE is a software package for the analysis of conserved genomic loci. Bioinformatics 32:786-788. DOI: 10.1093/bioinformatics/btv646. 
356 Faircloth BC., Branstetter MG., White ND., Brady SG. 2015. Target enrichment of

357

358

359

360

361

362

363

364

365

366

367

368

369

370

371

372

373

374

375

376

377

378

379

380

381

382

383

384 ultraconserved elements from arthropods provides a genomic perspective on relationships among Hymenoptera. Molecular Ecology Resources 15:489-501. DOI: 10.1111/17550998.12328.

Faircloth BC., McCormack JE., Crawford NG., Harvey MG., Brumfield RT., Glenn TC. 2012. Ultraconserved elements anchor thousands of genetic markers spanning multiple evolutionary timescales. Systematic Biology 61:717-26. DOI: 10.1093/sysbio/sys004.

Gnirke A., Melnikov A., Maguire J., Rogov P., LeProust EM., Brockman W., Fennell T., Giannoukos G., Fisher S., Russ C., Gabriel S., Jaffe DB., Lander ES., Nusbaum C. 2009. Solution hybrid selection with ultra-long oligonucleotides for massively parallel targeted sequencing. Nature Biotechnology 27:182-189. DOI: 10.1038/nbt.1523.

Grabherr MG., Haas BJ., Yassour M., Levin JZ., Thompson DA., Amit I., Adiconis X., Fan L., Raychowdhury R., Zeng Q., Chen Z., Mauceli E., Hacohen N., Gnirke A., Rhind N., di Palma F., Birren BW., Nusbaum C., Lindblad-Toh K., Friedman N., Regev A. 2011. Fulllength transcriptome assembly from RNA-Seq data without a reference genome. Nature Biotechnology 29:644-652. DOI: 10.1038/nbt.1883.

Haas BJ., Papanicolaou A., Yassour M., Grabherr M., Blood PD., Bowden J., Couger MB., Eccles D., Li B., Lieber M., MacManes MD., Ott M., Orvis J., Pochet N., Strozzi F., Weeks N., Westerman R., William T., Dewey CN., Henschel R., LeDuc RD., Friedman N., Regev A. 2013. De novo transcript sequence reconstruction from RNA-seq using the Trinity platform for reference generation and analysis. Nature Protocols 8:1494-1512. DOI: 10.1038/nprot.2013.084.

Harris RS. 2007. Improved pairwise alignment of genomic DNA. The Pennsylvania State University.

He D., Choi A., Pipatsrisawat K., Darwiche A., Eskin E. 2010. Optimal algorithms for haplotype assembly from whole-genome sequence data. Bioinformatics 26:i183-i190. DOI: 10.1093/bioinformatics/btq215.

Henderson AJ. 2011. A revision of Geonoma (Arecaceae).

Heyduk K., Trapnell DW., Barrett CF., Leebens-Mack J. 2016. Phylogenomic analyses of 
385

386

387

388

389

390

391

392

393

394

395

396

397

398

399

400

401

402

403

404

405

406

407

408

409

410

411

412

species relationships in the genus Sabal (Arecaceae) using targeted sequence capture. Biological Journal of the Linnean Society 117:106-120.

Hunt M., Newbold C., Berriman M., Otto TD. 2014. A comprehensive evaluation of assembly scaffolding tools. Genome Biology 15:R42. DOI: 10.1186/gb-2014-15-3-r42.

Kadlec M., Bellstedt DU., Le Maitre NC., Pirie MD. 2017. Targeted NGS for species level phylogenomics: "made to measure" or "one size fits all"? PeerJ 5:e3569. DOI: 10.7717/peerj.3569.

Li H., Durbin R. 2010. Fast and accurate long-read alignment with Burrows-Wheeler transform. Bioinformatics 26:589-595. DOI: 10.1093/bioinformatics/btp698.

Li H., Handsaker B., Wysoker A., Fennell T., Ruan J., Homer N., Marth G., Abecasis G., Durbin R. 2009. The Sequence Alignment/Map format and SAMtools. Bioinformatics 25:2078-9. DOI: 10.1093/bioinformatics/btp352.

McKenna A., Hanna M., Banks E., Sivachenko A., Cibulskis K., Kernytsky A., Garimella K., Altshuler D., Gabriel S., Daly M., DePristo MA. 2010. The Genome Analysis Toolkit: a MapReduce framework for analyzing next-generation DNA sequencing data. Genome research 20:1297-303. DOI: 10.1101/gr.107524.110.

Milne I., Stephen G., Bayer M., Cock PJA., Pritchard L., Cardle L., Shaw PD., Marshall D. 2013. Using Tablet for visual exploration of second-generation sequencing data. Briefings in Bioinformatics 14:193-202. DOI: 10.1093/bib/bbs012.

Reuter JA., Spacek D V., Snyder MP. 2015. High-throughput sequencing technologies. Molecular cell 58:586-97. DOI: 10.1016/j.molcel.2015.05.004.

Simpson JT., Wong K., Jackman SD., Schein JE., Jones SJM., Birol I. 2009. ABySS: a parallel assembler for short read sequence data. Genome Research 19:1117-23. DOI: 10.1101/gr.089532.108.

Smith BT., Harvey MG., Faircloth BC., Glenn TC., Brumfield RT. 2014a. Target Capture and Massively Parallel Sequencing of Ultraconserved Elements for Comparative Studies at Shallow Evolutionary Time Scales. Systematic Biology 63:83-95. DOI: 
413 Smith BT., McCormack JE., Cuervo AM., Hickerson MJ., Aleixo A., Cadena CD., Pérez-Emán 414 J., Burney CW., Xie X., Harvey MG., Faircloth BC., Glenn TC., Derryberry EP., Prejean J., 415 Fields S., Brumfield RT. 2014b. The drivers of tropical speciation. Nature 515:406-409. 416 DOI: $10.1038 /$ nature 13687.

417 


\section{Table $\mathbf{1}$ (on next page)}

Summary statistics for all samples, produced by SECAPR.

Reported for each sample are the number of sequencing reads in the FASTQ sequencing files, before (1. column) and after ( 2 . column) cleaning and trimming, the total count of assembled de novo contigs (3. column), the number of filtered contigs that matched target loci (4. column) and the number of sequencing reads that mapped to the new reference library generated from the contig MSAs during reference-based assembly (5. column). These summary statistics are automatically compiled and appended to a log file (summary_stats.txt) during different steps in the SECAPR pipeline. 
1 Table1: Summary statistics for all samples, produced by SECAPR. Reported for each sample are the number of sequencing reads

2 in the FASTQ sequencing files, before (1. column) and after (2. column) cleaning and trimming, the total count of assembled de novo

3 contigs (3. column), the number of filtered contigs that matched target loci (4. column) and the number of sequencing reads that

4 mapped to the new reference library generated from the contig MSAs during reference-based assembly (5. column). These summary

5 statistics are automatically compiled and appended to a log file (summary_stats.txt) during different steps in the SECAPR pipeline.

6

\begin{tabular}{|c|c|c|c|c|c|}
\hline $\begin{array}{l}\text { Sample } \\
\text { ID }\end{array}$ & $\begin{array}{c}\text { FASTQ read pairs } \\
\text { (raw) }\end{array}$ & $\begin{array}{c}\text { FASTQ read pairs } \\
\text { (cleaned) }\end{array}$ & Total contig count & Recovered target contigs & $\begin{array}{c}\text { Reads on target } \\
\text { regions }\end{array}$ \\
\hline 1087 & 291089 & 276072 & 277628 & 562 & 22308 \\
\hline 1086 & 244726 & 231326 & 230122 & 516 & 17969 \\
\hline 1140 & 206106 & 192676 & 153377 & 469 & 18039 \\
\hline 1083 & 377228 & 352646 & 309993 & 534 & 31922 \\
\hline 1082 & 277999 & 262378 & 258359 & 556 & 19491 \\
\hline 1085 & 307671 & 291377 & 309561 & 512 & 22030 \\
\hline 1079 & 315801 & 298450 & 306369 & 550 & 13969 \\
\hline 1061 & 209586 & 192407 & 177910 & 545 & 14474 \\
\hline 1068 & 295402 & 278069 & 264865 & 563 & 22013 \\
\hline 1063 & 354795 & 336356 & 356512 & 525 & 20439 \\
\hline 1080 & 459485 & 434951 & 433954 & 531 & 41068 \\
\hline 1065 & 217725 & 205290 & 204082 & 544 & 13524 \\
\hline 1073 & 302798 & 286021 & 289612 & 529 & 15598 \\
\hline 1070 & 295822 & 278011 & 295557 & 539 & 19288 \\
\hline 1064 & 408723 & 384908 & 405080 & 543 & 21531 \\
\hline 1074 & 408370 & 383604 & 398758 & 531 & 25476 \\
\hline 1166 & 405667 & 385442 & 410292 & 544 & 29697 \\
\hline
\end{tabular}




\section{Figure 1 (on next page)}

SECAPR analytical workflow.

The flowchart shows the basic SECAPR functions, which are separated into two separate steps (colored boxes). Blue box (1. reference library from raw data): in this step the raw reads are cleaned and assembled into contigs (de novo assembly); Orange box (2. reference based assembly with custom reference library): the contigs from the previous step are used for reference-based assembly, enabling allele phasing and additional quality control options, e.g. concerning read-coverage. Black boxes show SECAPR commands and white boxes represent the input and output data of the respective function. Boxes marked in grey represent multiple sequence alignments (MSAs) generated with SECAPR, which can be used for phylogenetic inference. 


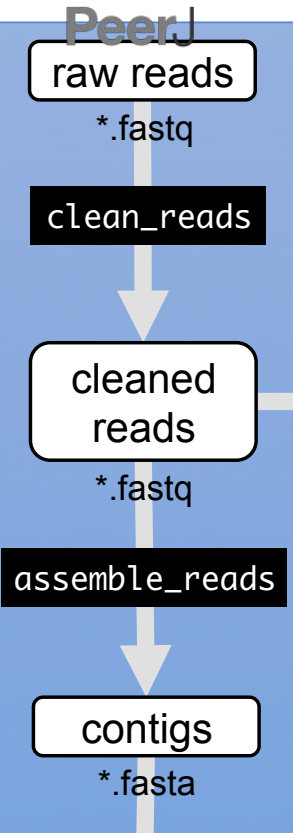

\section{Manuscript to ba revienend:}

secapr function

file content

*.format

\section{reference \\ assembly}

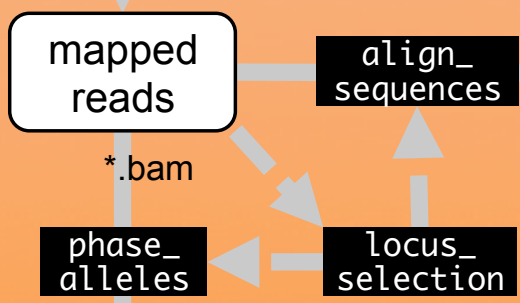

find_target_contigs

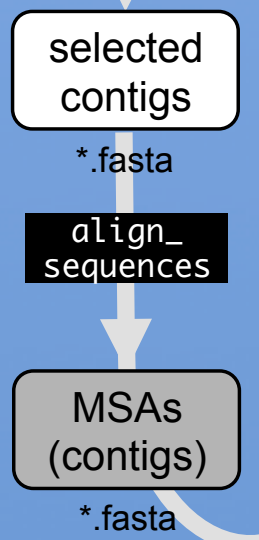

1. reference library

from raw data

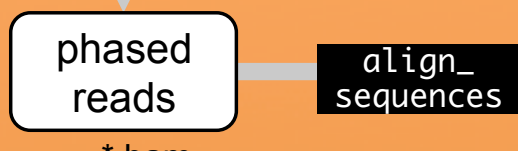

MSAs

(phased)

*.fasta

\section{2. reference based assembly with custom reference library}




\section{Figure 2}

Overview of FASTQc quality test result.

a) Before and b) after cleaning and adapter trimming of sequencing reads with the SECAPR function clean_reads. This plot, as produced by SECAPR, provides an overview of the complete dataset and helps to gauge if the chosen cleaning parameters are appropriate for the dataset. The summary plots show the FASTQc test results, divided into three categories: passed (green), warning (blue) and failed (red). The x-axis of all plots contains the eleven different quality tests (see legend). The bar-plots (left panels) represent the counts of each test result (pass, warning or fail) across all samples. The matrix plots (right panels) show the test result of each test for each sample individually (y-axis). This information can be used to evaluate both, which specific parameters need to be adjusted and which samples are the most problematic. 

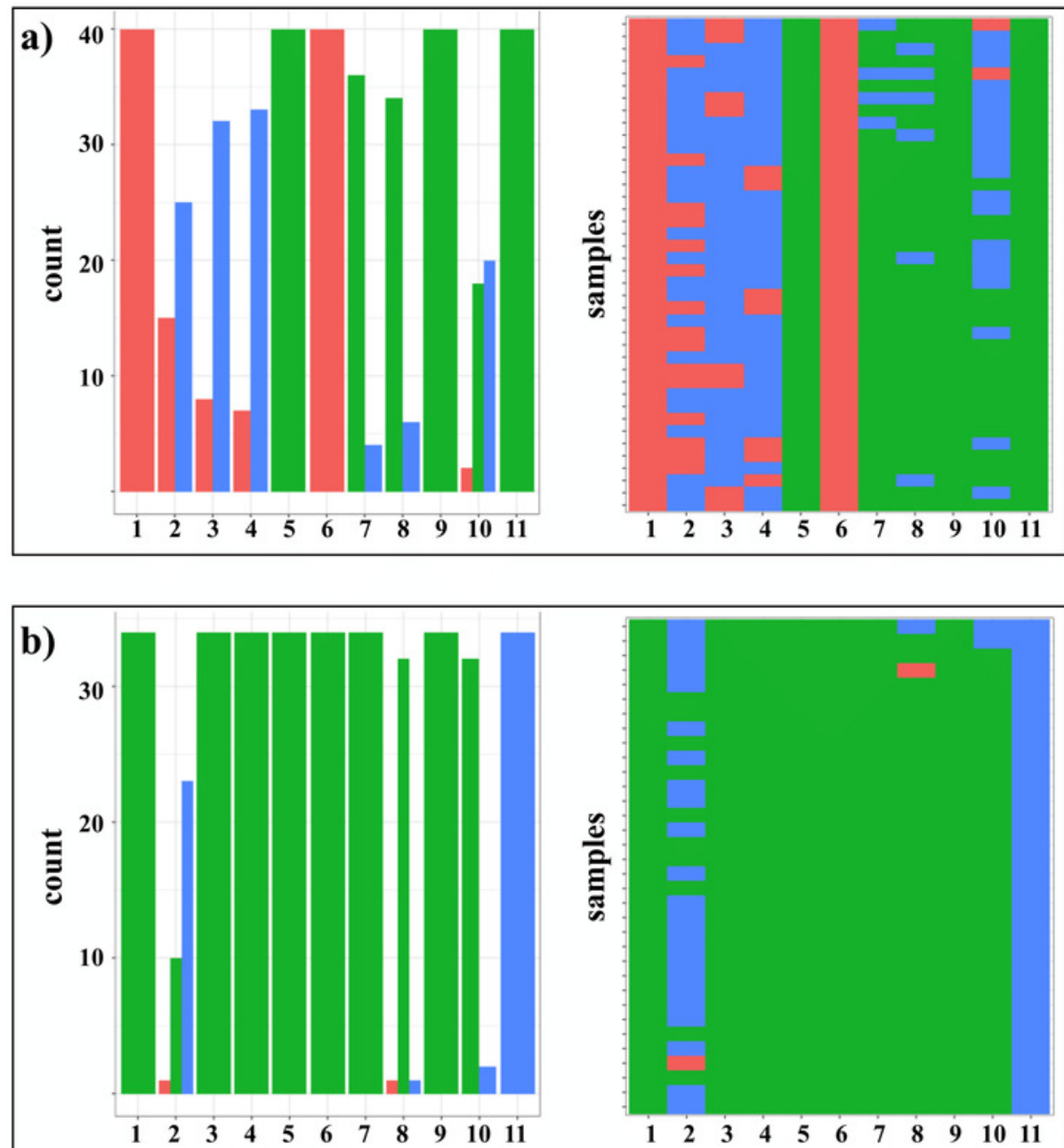

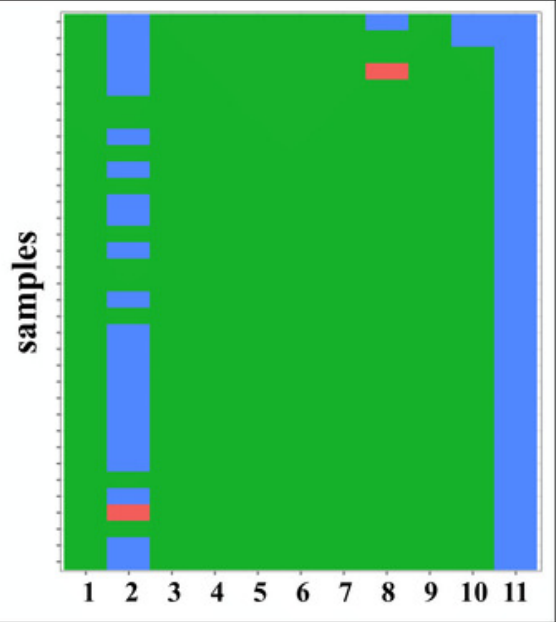

Test result:

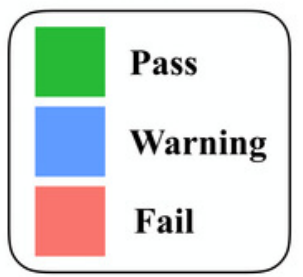

Test index:

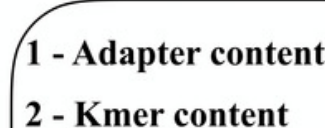

3 - Overrepresented sequences

4 - Per base sequence content

5 - Per base $N$ content

6 - Per base sequence quality

7 - Per tile sequence quality

8 - Sequence duplication levels

9 - Per sequence GC content

10 - Per sequence quality scores

11 - Sequence length distribution 


\section{Figure 3}

Reference-based assembly including heterozygous sites.

BAM-assembly file as generated with the SECAPR reference_assembly function, shown exemplarily for one exon locus (1/837) of one of the Geonoma samples (1/17). The displayed assembly contains all FASTQ sequencing reads that could be mapped to the reference sequence (top panel). The reference sequence in this case is the de-novo contig that was matched to the reference exon 'Elaeis 1064 3'. DNA bases are color-coded (A - green, C blue, $\mathrm{G}$ - black, $\mathrm{T}$ - red). The enlarged section contains a heterozygous site, which likely represents allelic variation, as both variants $A$ and $G$ are found at approximately equal ratio. 


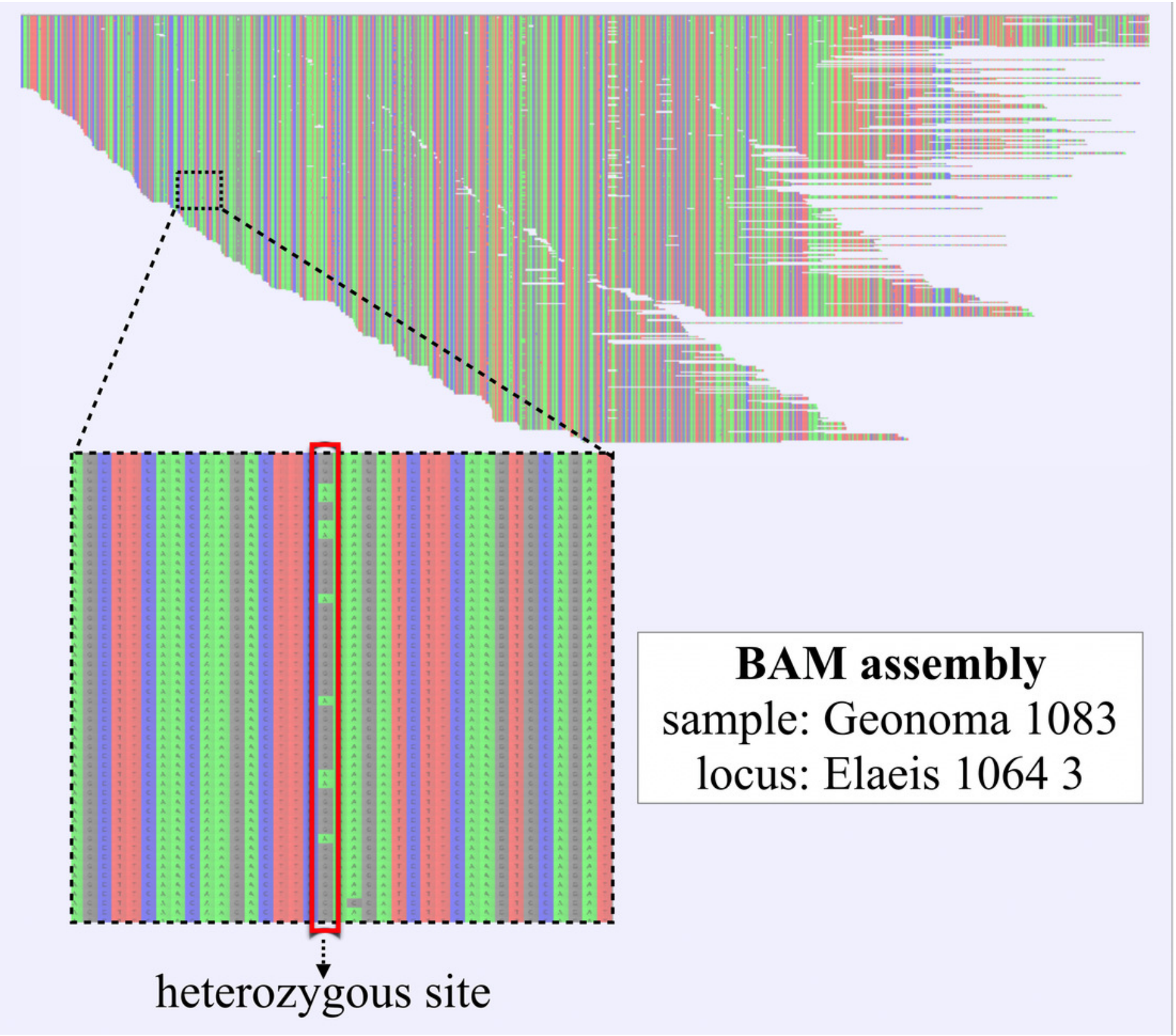




\section{Figure 4}

Overview of sequence yield for Geonoma sample data, produced with SECAPR.

The matrix plots show an overview of the contig yield and read-coverage for all targeted loci $(a+b)$ and for the selection of the 50 loci with the best read coverage $(c+d)$, selected with the SECAPR function locus_selection (see Supplementary Table S5 for loci-names corresponding to indices on X-axes). a) and c) show if de novo contigs could be assembled (blue) or not (white) for the respective locus (column) and sample (row). Contig MSAs were generated for all loci that could be recovered for at least 3 samples (center row - green). b) and d) show the read coverage (see legend) for each exon locus after reference-based assembly. The reference library for the assembly consisted of the consensus sequences of each contig MSA, and hence is genus specific for Geonoma.

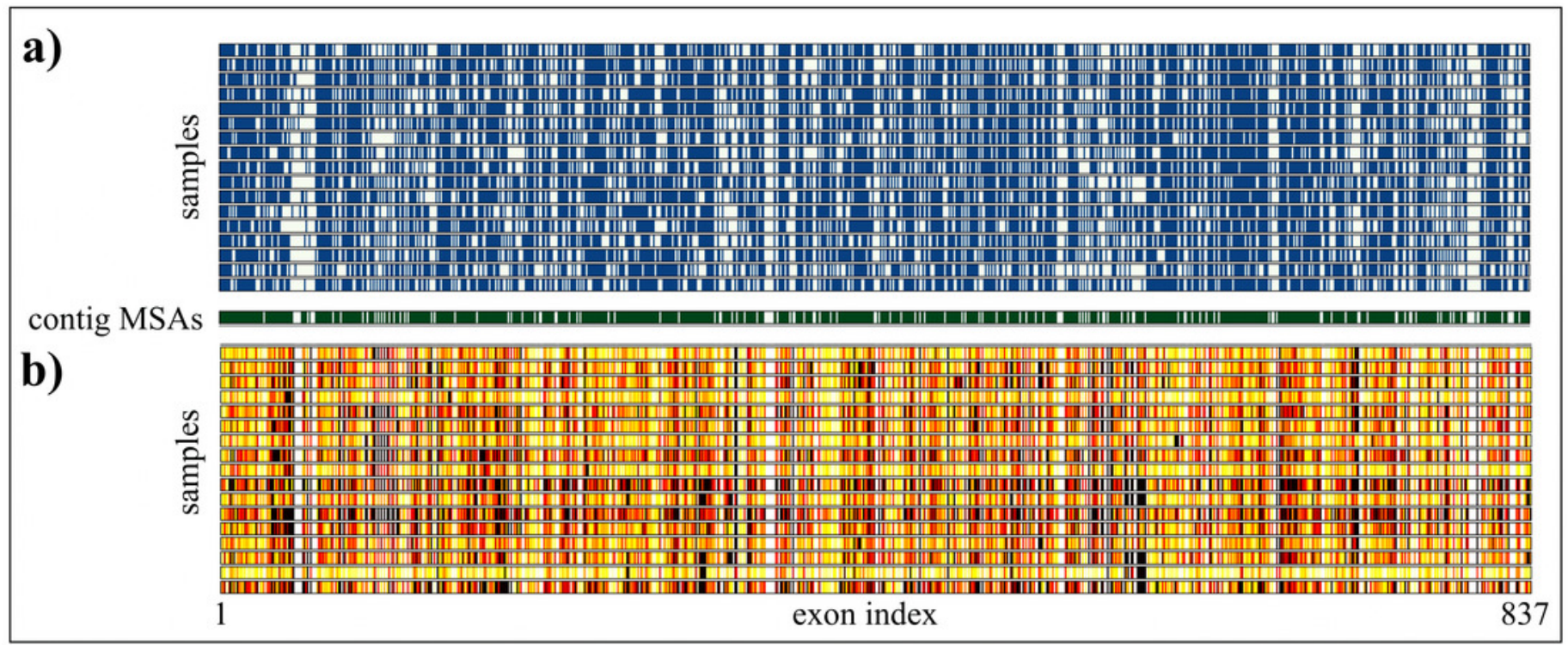

c)

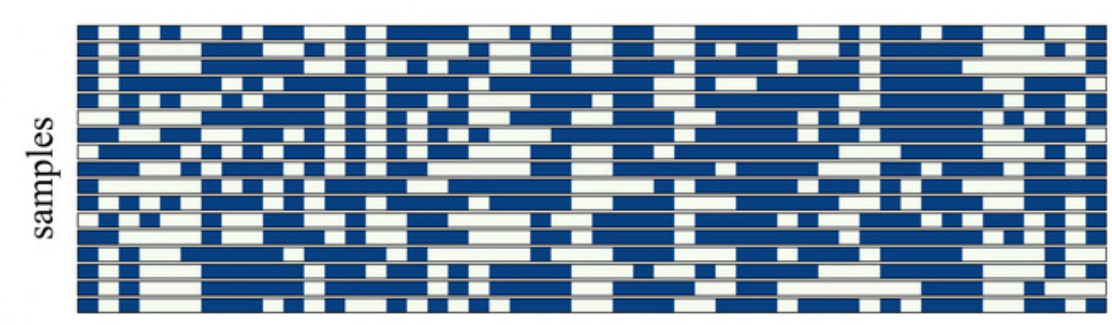

contig MSAs

d)
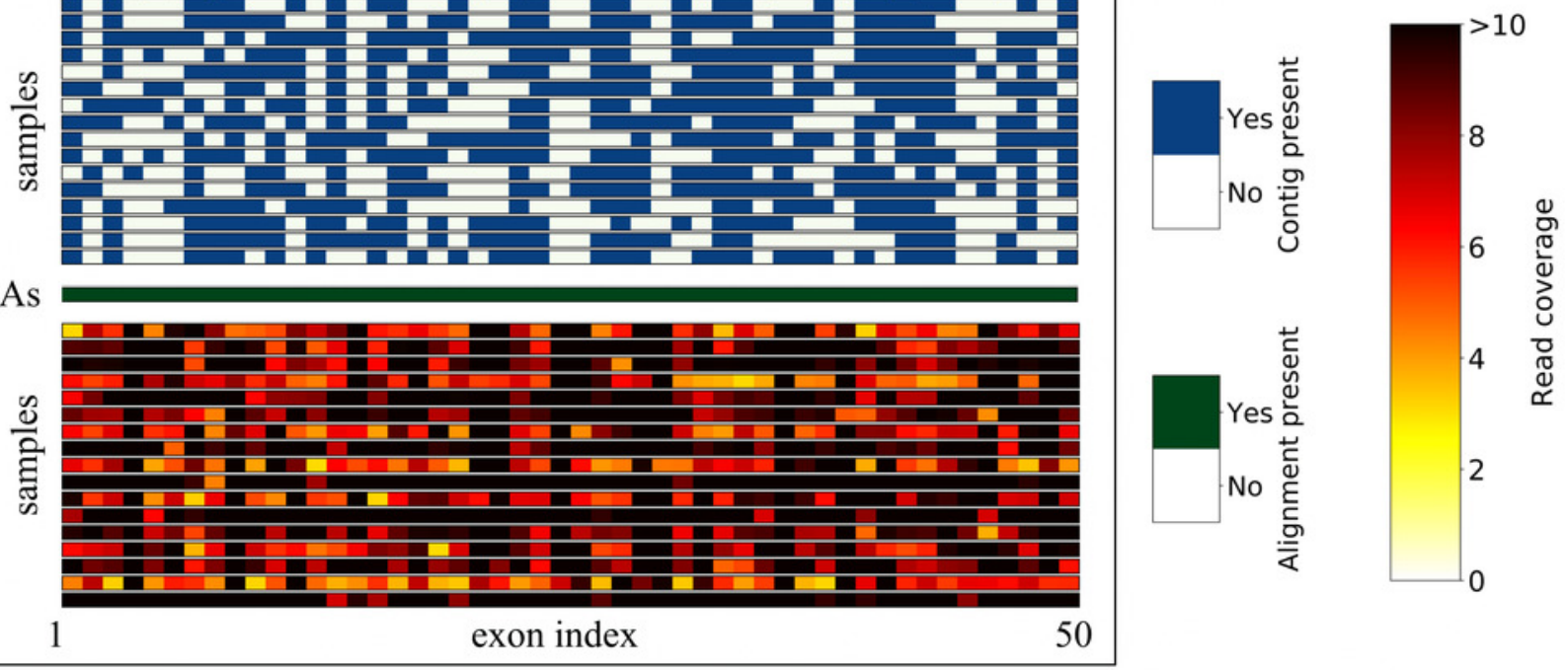